\title{
Gaia and the asteroids: Local test of GR
}

\section{Daniel Hestroffer ${ }^{1}$ and S. Mouret ${ }^{1,2}$ and F. Mignard ${ }^{3}$ and P. Tanga ${ }^{3}$ and J. Berthier ${ }^{1}$}

${ }^{1}$ IMCCE, Observatoire de Paris, CNRS,

77 Av. Denfert-Rochereau F-75014 Paris, France

email: hestro@imcce.fr, berthier@imcce.fr

${ }^{2}$ Lohrman Observatory, Dresden, Germany; email: serge.mouret@tu-dresden.de

${ }^{3}$ Cassiopée, Observatoire de la Cote d'Azur, CNRS, Mont-Gros F-06300 Nice, France email: mignard@oca.eu, tanga@oca.eu

\begin{abstract}
We present in the following some capabilities of the Gaia mission for performing local test of General Relativity (GR) based on the astrometry of asteroids. This ESA cornerstone mission, to be launched in Spring 2012, will observe - in addition to the stars and QSOs - a large number of small solar system bodies with unprecedented photometric and, mostly, astrometric precisions. Indeed, it is expected that about 250,000 asteroids will be observed with a nominal precision ranging from a few milli-arcsecond (mas), to sub-mas precision, depending on the target's brightness. While the majority of this sample is constituted of known main-belt asteroids orbiting between Mars and Jupiter, a substantial fraction will be made of near-Earth objects, and possibly some newly discovered inner-Earth or co-orbital objects.

Here we show the results obtained from a simulation of Gaia observations for local tests of GR in the gravitational field of the Sun. The simulation takes into account the time sequences and geometry of the observations that are particular to Gaia observations of solar system objects, as well as the instrument sensitivity and photon noise. We show the results from a variance analysis for the nominal precision of the joint determination of the solar quadrupole $J_{2}$ and the PPN parameter $\beta$. Additionally we include the link of the dynamical reference frame to the conventional kinematically non-rotating reference frame (as obtained in the visible wavelength by Gaia observations of QSOs). The study is completed by the determination of a possible variation of the gravitational constant $\dot{G} / G$, and deviation from Newtonian $1 / r^{2}$ gravitational law. Comparisons to the results obtained from other techniques are also given.
\end{abstract}

Keywords. Gaia, asteroids, astrometry, GR, solar quadrupole, reference frame

\section{Introduction}

One century almost after the appearance of Albert Einstein's "Allgemeine Relativitätstheorie" paper (1915), the theory of general relativity (GR) is still the subject of debates as alternative to the metric theories have been proposed, and tests of the GR in particular in the parameterized post-Newtonian (PPN) frame can still be undertaken (e.g. Will 2006). After the first experiment of Einstein with the perihelion drift of Mercury, Gilvarry (1953) or Dicke (1965) noted that near-Earth asteroids with large eccentricity as (1566) Icarus are also good candidates for such local tests. Past efforts to test the theory of GR with the asteroid Icarus and other solar system objects revealed however unsuccessful (Shapiro et al. 1971) or could provide a test at the percent level (Sitarski 1992, Zhan 1994), mainly because of the many systematic or large stochastic errors in the observations, as well as in the dynamical model itself. For instance it was basically uneasy to disentangle relativistic effects from possible unknown non-gravitational effects. With the advent of modern high precision astrometry, from ground-based radar observations (Ostro 2007), or from space with Hipparcos (Hestroffer et al. 1998) and Gaia 
(Mignard et al. 2007), the situation will change drastically and asteroids should be potentially powerful targets as is the Moon from LLR ranging, Mercury with Beppi-Colombo, or other planets with radar ranging. There are some advantages to consider asteroids: they are numerous; probing a wide range in distance in the Solar System ${ }^{a}$; and they are also small in size and mass, making them behave dynamically as free test particles with little shape effects on their astrometry. On another hand, their fast motion and faintness make them difficult to observe with small telescopes ${ }^{b}$, and last, non-gravitational effects by perturbing their orbits can mimic some of the foreseen relativistic effects.

We present in the following an analysis of the performance that could be achieved from the astrometry of Gaia asteroids, and comparison to other works. First the mission and instruments characteristics are briefly presented. We then give the formal precision for the determination of the PPN parameter $\beta$ together with the solar quadrupole $J_{2}$, a possible variation of the constant of gravitation $\dot{G} / G$, a possible deviation from the Newtonian force $\left(1 / r^{2}+\kappa\right)$, and last the precision that will be achieved for linking the dynamical reference frame to the kinematical optical-ICRF that will be obtained with Gaia.

\section{The Gaia mission}

The astrometric Gaia mission will regularly scan the whole celestial sphere down to magnitude $V \leqslant 20$, providing high precision data for a huge number of celestial objects (Lindegren 2009); including not only stars but also solar system objects, mostly asteroids. Compared to its precursor Hipparcos, Gaia will provide a wealth of information on asteroids: much higher precision in astrometry and spectro-photometry, for a number of targets about 4 orders of magnitude larger. In particular Gaia will observe NEOs down to low solar elongation (see Table 1). One can estimate that about 250,000 asteroids will be regularly observed. The average number of observations given here for an MBA can be smaller for a NEO or for an object close to the magnitude limit. The high precision astrometry (at sub-mas level precision) that will be acquired for asteroids should allow to revise the test of GR from analysing their orbit. The present work can be separated in two steps: simulation of the observations for the targets, and variance analysis for the various parameter estimation.

Based on the principle of Hipparcos for global astrometry, the Gaia telescope will not allow a pointing observation of an object. Instead, the target is observed when it is transiting the field-of-view (FOV). We have thus performed a simulation of the observations taking into account the time sequences and geometry of the observations that are particular to Gaia observations of solar system objects, as well as the instrument sensitivity and photon noise. We have also considered two sets of object populations. The first set includes all known asteroids, the second one consists of a population of synthetic NEOs. Indeed, not all NEOs larger than $\approx 500 \mathrm{~m}$ have been discovered yet, but many will be known at the time Gaia will operate from current or future ground-based surveys. Among this second set, several synthetic populations have been constructed combining random brightness and orbital elements following the de-biased distributions from Bottke et al. (2002). The second step of simulations in the chain gives an estimation of the astrometric precision as a function of the object size, motion and brightness. As given in Table 1,

${ }^{a}$ Going from near-Earth objects - NEO, main-belt asteroids - MBA, Jupiter Trojans, Centaurs and trans-Neptunian objects - TNO, or long-period comets - LPC.

${ }^{b}$ High precision optical astrometry generally necessitates a very good astrometric catalogue free as possible of any zonal error. Radar echo can be obtained only for the closest NEOs. 
the formal precision ${ }^{c}$ of one astrometric point in the highest resolution direction varies between 0.3 to 5 mas (milli-arcsecond) and mainly depends on the source brightness.

Table 1. General figure of the Gaia mission and observations of Solar System Objects.

\begin{tabular}{ll}
\hline Launch date / duration & spring $2012 / 5$ years \\
Celestial sphere coverage & 6 month \\
Limiting magnitude / size & $V \leqslant 20 / \approx 250$ mas \\
Number of stars & $\approx 10^{9}$ \\
Number of asteroids /NEOs & $\approx 250.000 / \approx 2500$ \\
Number of observations & $\approx 60$ transits/target \\
Solar elongation & $45^{\circ} \leqslant L \leqslant 135^{\circ}$ \\
Astrometry $(\mathrm{CCD})$ & $\mathrm{AL}: 0.3-5 \mathrm{mas}$ \\
& $\mathrm{AC}: 6-12 \times \mathrm{AL}$ \\
Photometry $(\mathrm{CCD})$ & $0.001-0.05 \mathrm{mag}$ \\
\hline
\end{tabular}

\section{Global parameters determination}

Starting from the simulated data of the previous section, we can perform a variance analysis for various parameters to be estimated (or adjusted), in particular global parameters common to all, or a large subset of targets. Since the orbits are already known and all foreseen parameters are small, we can linearise our system of observational equations and solve it by least squares. The vector of unknown parameters $d \mathbf{p}=\left(d \mathbf{q}_{i} ; d \mathbf{q}_{g}\right)$ will contain the correction to the initial conditions $d \mathbf{q}_{i}$ specific to each asteroid $i$ and the global parameters $d \mathbf{q}_{g}$ common to all objects. The matrix of partial derivatives:

$$
[\partial \mathbf{x} / \partial \mathbf{p}]_{i}=\left[\partial x / \partial q_{i} ; \partial x / \partial q_{g}\right] \equiv\left[\mathbf{B}_{i} ; \mathbf{A}_{i}\right]
$$

yields the variation of the target's position at time of observation, and is computed numerically. The variance of the global parameters is then obtained from the inversion of a reduced normal matrix (Söderhjelm \& Lindegren 1982) :

$$
\mathbf{U}=\sum_{i}\left(\mathbf{A}_{i}^{\prime} \cdot \mathbf{A}_{i}\right)-\mathbf{A}_{i}^{\prime} \cdot \mathbf{B}_{i}\left(\mathbf{B}_{i}^{\prime} \cdot \mathbf{B}_{i}\right)^{-1} \mathbf{B}_{i}^{\prime} \cdot \mathbf{A}_{i} \quad ; \quad \operatorname{var}\left(d \mathbf{q}_{g}\right)=\sigma_{o} \mathbf{U}^{-1}
$$

where matrices $\left(\mathbf{B}_{i}^{\prime} \cdot \mathbf{B}_{i}\right)$ are of dimension $6 \times 6$ as the state vector of the asteroid, and $\left(\mathbf{A}_{i}^{\prime} . \mathbf{A}_{i}\right)$ of dimension $n_{g} \times n_{g}$, i.e. the number of global parameters, no more than a dozen.

The global parameters that can be estimated are the mass of perturbing asteroids (Mouret et al. 2007) or other parameters that would affect all orbits such as the PPN parameter $\beta$, the solar $J_{2}$. It is well known that both the Sun quadrupole $J_{2}$ and GR imply an advance of the perihelion of the orbit that can hardly be separated from the observation of one single target, such as Mercury alone. Indeed, putting $m_{\odot}=G M_{\odot} / c^{2}$ the secular drift ${ }^{d}$ of the orbit's argument of periapsis is given by :

$$
\Delta \omega=\Delta \omega_{\mid P P N}+\Delta \omega_{\mid J_{2}}
$$

${ }^{c}$ A typical star will cross 9 CCDs in a row during one FOV transit, but a fast moving object will not; thus, in order to be more conservative we consider that one transit is reduced to one single CCD exposure. Note that the typical $20 \mu$ as precision found in the literature for a star is much smaller because it moreover combines all $\approx 100$ transits gathered over the mission.

${ }^{d}$ Linear part of the orbital elements variation after periodic terms have been averaged, and also after the larger-but well known - secular perturbations of the planets have been removed. 
Table 2. Overview of the expected results for the local test of GR with Gaia and the asteroids, and comparison to other experiments in the solar system. (Check mark means parameter determination is possible but not explicitly provided.)

\begin{tabular}{|c|c|c|c|c|c|c|c|c|}
\hline & $\begin{array}{c}\sigma(\beta) \\
-\end{array}$ & $\begin{array}{c}\sigma\left(J_{2}\right) \\
-\end{array}$ & $\begin{array}{c}\text { Correl } \\
-\end{array}$ & $\begin{array}{c}\sigma(\dot{G} / G) \\
{\left[\mathrm{yr}^{-1}\right]}\end{array}$ & $\begin{array}{c}\sigma(\kappa) \\
{\left[\mathrm{m} . \mathrm{s}^{-2}\right]}\end{array}$ & $\begin{array}{c}\sigma\left(W_{o}\right) \\
{[\mu \mathrm{as}]}\end{array}$ & $\begin{array}{c}\sigma(\dot{W}) \\
{\left[\mu \text { as.yr }^{-1}\right]}\end{array}$ & Ref. \\
\hline Gaia & $0.6-6 \times 10^{-4}$ & $0.5-10 \times 10^{-8}$ & $0.1-0.9$ & $2 \times 10^{-12}$ & $8 \times 10^{-11}$ & {$[5-5-15]$} & {$[1-1-5]$} & \\
\hline $\mathrm{LLR}^{1,2}$ & $1.1 \times 10^{-4}$ & - & - & $3 \times 10^{-13}$ & - & - & $-\quad$ & {$[1]$} \\
\hline Ephemeris ${ }^{2,3}$ & $2 \times 10^{-4}$ & assumed & - & $5 \times 10^{-13}$ & $\sqrt{ }$ & $\sqrt{ }$ & 40 & {$[2]$} \\
\hline Bepi Colombo ${ }^{1}$ & $2 \times 10^{-6}$ & $2 \times 10^{-9}$ & 0.997 & $\sqrt{ }$ & - & - & - & {$[3]$} \\
\hline NEOs radar ${ }^{4}$ & $\sqrt{ }$ & $\sqrt{ }$ & $\sqrt{ }$ & - & - & - & - & {$[4]$} \\
\hline $\mathrm{TNOs}^{5}$ & - & - & - & - & $1.6 \times 10^{-10}$ & - & - & {$[5]$} \\
\hline
\end{tabular}

Notes:

${ }^{1}$ In the LLR technique as well as for the Bepi-Colombo experiment, the PPN $\beta$ is derived from Nordvedt $\eta_{\mathrm{N}}=4 \beta-\gamma-3$ parameter. Also $\gamma$ is hence assumed to be know with sufficient accuracy.

${ }^{2}$ Precision on $\dot{G} / G$ is improving rapidly with time and increased data span. The same is true for the pulsar timing technique (Deller et al. 2008).

${ }^{3}$ Based on model value for the Solar $J_{2}$ in Fienga et al. (2008), Pitjeva $(2005) ; \sigma\left(J_{2}\right)=3 \times 10^{-8}$ in Pitjeva (2009).

${ }^{4}$ Radar measurements will provide these parameters from a set of $\approx 20$ observed targets.

${ }^{5}$ Based on analysis of $\approx 25$ TNOs, not directly comparable to the Gaia value (see text).

Ref.: [1] Williams \& Folkner (2009) ; [2] Folkner (2009) for $\dot{G}, \kappa$ and $W$ Pitjeva (2009), Fienga et al. (2008) for $\beta, J_{2}$ and $\dot{G}$; [3] Milani (2009) ; [4] Margot \& Giorgini (2009) ; [5] Wallin et al. (2007).

$$
=\frac{3 m_{\odot}}{a\left(1-e^{2}\right)}\left[\frac{2+2 \gamma-\beta}{3}+\frac{R_{\odot}^{2}}{4 a m_{\odot}} \frac{\left(5 \cos ^{2} i-1\right)}{\left(1-e^{2}\right)} J_{2}\right] n\left(t-t_{0}\right)
$$

while the other elliptical elements, in particular the drift for the longitude of the node, is driven by the Sun quadrupole only $\left(\Delta \Omega=\Delta \Omega_{\mid J_{2}}\right)$. Here one readily sees that the relativistic and $J_{2}$ secular effects - being large for high eccentricities - act differently through the asteroids ${ }^{e}$ inclination $i$ and mostly through its semi-major axis $a$ and eccentricity $e$. Other parameters of the dynamical model that can similarly be estimated are a possible time-variation of the gravitational constant $\dot{G}$, a violation of the Newtonian $1 / r^{2}$ law of gravitation ${ }^{f}$, or a rotation ${ }^{h} \mathbf{W}=\mathbf{W}_{o}+\mathbf{W}_{1}\left(t-t_{0}\right)$ between the kinematically or dynamically non-rotating frames associated to either the QSOs or the ephemerides, respectively.

\section{Results - Discussion}

Solving for the global system (3.2) yields the formal precision given in Table 2 . The basic output will be the derivation of the solar $J_{2}$ and PPN $\beta$ with no model assumption for the Sun interior, shape or rotation. The precision is not better than what is achieved today from LLR data, but yet independent of the Nordvedt $\eta_{\mathrm{N}}$ parameter. Note that the parameter $\gamma$ in Eq. (3.3) is known with much better accuracy (from Gaia itself, Mignard (2009), Hobbs et al. (2009), or other experiments). The formal precision and correlation are nevertheless sensitive by roughly one order of magnitude to the actual number of targets brighter than magnitude $V \leqslant 20$, to the actual number of observations per target, and to the observations of highly eccentric objects orbiting close from the Sun.

${ }^{e}$ In the case of major planets most eccentricities and inclination are small, and similar.

$f$ One includes an additional acceleration term in the equation of motion in a way similar to Wallin et al. (2007), but here systematically at any distance to the Sun, $\ddot{\mathbf{r}}=-G M_{\odot}\left(1 / r^{3}+\kappa\right) \mathbf{r}$.

${ }^{h}$ That is the sum of a constant rigid rotation $\mathbf{W}_{o}$ at reference epoch $t_{0}$ and a rotation rate $\mathbf{W}_{1}$. 
One will be able to measure a possible variation $\dot{G} / G$ at the $10^{-12} \mathrm{yr}^{-1}$ level, and validate the Newtonian $1 / r^{2}$ law in the main belt of asteroids at the $10^{-10}$ level. Note a fundamental difference with the 'Pioneer-anomaly' kind of test, which consider a deviation to the Newtonian law triggered only at some given distance to the Sun, e.g. 5, 10 or $20 \mathrm{AU}$. If we restrict the analysis to the few Centaurs and TNOs observed by Gaia alone over five years, the precision $\sigma(\kappa)$ drops to $6 \times 10^{-7} \mathrm{~m} / \mathrm{s}^{2}$. Note also that a possible spatial variation of $G$ is not considered, and that the time variation is coupled with the mass-loss of the Sun, i.e. what is actually measured is $d(G M) / d t$. One will also be able to measure a possible rotation rate $\mathbf{W}_{1}$ to the order of $6 \mu \mathrm{as} / \mathrm{yr}$ $\left(\approx 3 \times 10^{-11} \mathrm{rad} / \mathrm{yr}\right)$ between the dynamical and kinematical reference frames. This is still far much larger than the geodetic precession of the Solar System orbiting around the Galaxy $\dot{\Omega}_{G P}=3 / 2\left(V_{\odot} / c\right)\left(G M_{g} / c / R_{\odot}^{2}\right) \approx 0.02 \mu \mathrm{as} / \mathrm{yr}$, or current bounds to a Gödelian rotation of the Universe $\left(|\omega| \leqslant 10^{-2} \mu \mathrm{as} / \mathrm{yr}\right)$. This formal precision is moreover to be balanced by the precision with which the materialization of the kinematically non-rotating frame can be achieved (Lindegren 2009, Zharov et al. 2009) and the systematic errors that can enter in the process of asteroids orbit fitting. Another relativistic effect that will perturb the orbits is the gravitomagnetic Lense-Thirring effect from the spinning Sun. Putting $J_{\odot}=\frac{4}{5} M_{\odot} R^{2} \omega_{\odot}$ where $\omega_{\odot}$ is the spin rate of the Sun, the precession of the orbit are $\dot{\Omega}_{L T}=2 \frac{G}{c^{2}} \frac{J_{\odot}}{a^{3}\left(1-e^{2}\right)^{3 / 2}}$ for the node and $\dot{\varpi}_{L T}=-\frac{G}{c^{2}} \frac{J_{\odot}\left(1-3 \sin ^{2} i / 2\right)}{a^{3}\left(1-e^{2}\right)^{3 / 2}}$ for the longitude of the periapsis and the mean anomaly. The Lense-Thirring perturbation can be of the order of 10 to $100 \mu \mathrm{as} / \mathrm{yr}$, depending on the target orbit, and must hence be taken into account.

Combining the radar data already acquired by Margot \& Giorgini (2009), which are of comparable quality and moreover orthogonal in essence by providing the range of the target, to the Gaia data when available, should also improve these numbers and possibly provide one of the best (direct) measure of the PPN $\beta$. Possible test of the Strong Equivalence Principle from analysis of the asteroids orbits (in particular the Trojans around the stable Lagrangian points of Jupiter, Orellana \& Vucetich (1988)), influence of non-gravitational effects, and derivation of the orbits precession from the Lense-Thirring effect are under study.

\section{References}

Bottke, W. F., Morbidelli, A., Jedicke, R., et al. 2002, Icarus, 156, 399

Deller, A. T., Verbiest, J. P. W., Tingay, S. J., \& Bailes, M. 2008, ApJL, 685, L67

Dicke, R. H. 1965, AJ, 70, 395

Fienga, A., Manche, H., Laskar, J., \& Gastineau, M. 2008, A\&A, 477, 315

Folkner, W. M. 2009, this proceedings, 155. BAAS, 41, \#06.01

Gilvarry, J. J. 1953, Physical Review, 89, 1046

Hestroffer, D., Morando, B., Hog, E., et al. 1998, A\&A, 334, 325

Hobbs, D., Holl, B., Lindegren, L., et al. 2009, this proceedings, 315. BAAS, 41, \#16.03

Lindegren, L. 2009, this proceedings. BAAS, 41, \#16.01

Margot, J.-L. \& Giorgini, J. D. 2009, this proceedings, 183. BAAS, 41, \#07.01

Mignard, F. 2009, this proceedings, 306. BAAS, 41, \#16.02

Mignard, F., Cellino, A., Muinonen, K., et al. 2007, Earth Moon and Planets, 101, 97

Milani, A. 2009, this proceedings, 356. BAAS, 41, \#18.01

Mouret, S., Hestroffer, D., \& Mignard, F. 2007, A\&A, 472, 1017

Orellana, R. B. \& Vucetich, H. 1988, A\&A, 200, 248

Ostro, S. J. and Giorgini, J. D. and Benner, L. A. M. 2007, IAU Symposium \#236, 143

Pitjeva, E. V. 2005, Astronomy Letters, 31, 340-349 
Pitjeva, E. V. 2009, this proceedings, 170. BAAS, 41, \#06.03

Shapiro, I. I., Smith, W. B., Ash, M. E., \& Herrick, S. 1971, AJ, 76, 588

Sitarski, G. 1992, AJ, 104, 1226

Söderhjelm, S. \& Lindegren, L. 1982, A\&A, 110, 156

Wallin, J. F., Dixon, D. S., \& Page, G. L. 2007, ApJ, 666, 1296

Will, C. M. 2006, Living Reviews in Relativity, 9

Williams, J. G. \& Folkner, W. M. 2009, this proceedings. BAAS, 41, \#08.01

Zhang, J.-X. 1994, Chinese Astron. and Astroph., 18, 108-115

Zharov, V., Sazhin, M., Sementsov, V., Kuimov, K., \& Sazhina, O. 2009, this proceedings, 50. BAAS, 41, \#02.06 